\title{
Rationale and Design of a Study to Test the Effect of Personal Protective Aids on Hypertension and Diabetes in People Living with High Levels of Air Pollution (Clinicaltrials.Gov Identifier NCT04854187)
}

Dweep Barbhaya ( $\nabla$ dweepbarbhaya@gmail.com )

Medstar Washington Hospital Center https://orcid.org/0000-0003-1934-0913

Jennifer Tran

Medstar Washington Hospital Center

Aditya Khetan

Hamilton Health Sciences

Vittal Hejjaji

Saint Luke's Mid America Heart Institute

\section{Supreme Jain}

Indian Institute of Technology Kanpur

Chee Chan

Medstar Washington Hospital Center

Anubha Goel

Indian Institute of Technology Kanpur

\section{Methodology}

Keywords: Air Pollution, Personal protective device, Hypertension, Diabetes

Posted Date: July 21st, 2021

DOl: https://doi.org/10.21203/rs.3.rs-719305/v1

License: (c) (1) This work is licensed under a Creative Commons Attribution 4.0 International License.

Read Full License 


\section{Abstract}

Background:

Air pollution is the largest environmental cause of disease and premature death in the world today, disproportionally affecting low-middle-income countries(LMIC) such as India. Studies have shown that exposure to particulate matter $<2.5 \mu \mathrm{m}$ (PM2.5) can contribute to cardiovascular disease and increase mortality. We hypothesize that the use of personal protective aids (home indoor air-purifiers/N-95 masks) can decrease systolic blood pressure(SBP) in people with hypertension and decrease fasting blood glucose(FBG) in those with diabetes.

Method:

This is a prospective randomized cross-over study in Dalkhola, India- an area of high ambient PM2.5 levels. Participants between 18-70 years of age with hypertension $(n=130)$ and diabetes $(n=33)$ will be invited to participate in the study. They will be randomized to either an intervention or control arm for 4 weeks, after which they will cross over to the other arm following a 2-week washout period. The intervention will consist of using an indoor air-purifier at night and N-95 mask when outdoors. The control period will involve using an identical air purifier and N-95 mask, with the filter removed (sham filtration). Participants and outcome assessors will be blinded to study arm assignment.

Outcome evaluation:

The primary outcome of the study is the absolute reduction in SBP among people with hypertension and absolute reduction in FBG among people with diabetes.

Discussion:

This is the first randomized controlled trial to evaluate the use of personal protective aids as a therapeutic measure in people with hypertension and diabetes exposed to high levels of PM2.5. Given the high burden of air pollution in LMICs, there is an urgent need for adaptation measures targeting people at high risk for mortality from this exposure. The results of our study will demonstrate if personal protective aids can be a viable adaptation measure for people living with hypertension and diabetes in areas with a high burden of air pollution.

\section{Introduction:}

Pollution is the largest environmental cause of disease and premature death in the world today (1). Nearly $92 \%$ of pollution-related deaths occur in low and middle-income countries such as India. Diseases caused by pollution are most prevalent amongst the marginalized sections of society, at every country income level (2). Although more than $70 \%$ of diseases caused by pollution are non-communicable diseases, such as myocardial infarction and stroke, strategies to decrease morbidity and mortality from pollution at an individual level are lacking (3). 
Ambient noise and air pollution (fine particulate matter $<2.5 \mu \mathrm{m}-\mathrm{PM} 2.5$ ) represent the two most important environmental risk factors. Together, they contribute to over $75 \%$ of the disease and disability burden associated with known environmental risk factors, most importantly cardiovascular (CV) morbidity and mortality (4). Short-term elevations in PM2.5 increase the relative risk of acute CV events by $1-3 \%$ within a few days. Longer-term exposures over several years increase this risk by a larger magnitude $(\sim 10 \%)$, which is partially attributable to the development of cardiometabolic conditions such as hypertension and diabetes mellitus. As such, ambient PM2.5 poses a major threat to global public health $(5,6)$. India, a rapidly developing country, is one of the most heavily polluted countries in the world and also has one of the highest burden of non-communicable diseases(7). While strategies to decrease air pollution primarily occur at a government and societal level, it will likely be years, if not decades, before individuals will see the effects from these policies and exposure to air pollutants is below thresholds that increase risk of adverse cardiovascular outcomes. Given this, urgent strategies are needed

to decrease the health effects of high air pollution at an individual level, especially in people at elevated cardiovascular risk.

Portable or central home air- filtration systems have been shown to reduce indoor PM2.5 levels by 50$60 \%(8,9)$. Preliminary data shows that the use of personal protective aids (such as home air purifiers and $\mathrm{N}-95$ masks) can decrease systolic blood pressure and blood glucose in healthy individuals exposed to high levels of air pollution (8-14). A considerable body of evidence suggests that the environmental agents, such as PM2.5, induce low-grade inflammation, oxidative stress, vascular dysfunction, and autonomic nervous system imbalance, thereby facilitating the development of diseases such as hypertension and diabetes $(15,16)$. Through their impact on traditional risk factors and via additional mechanisms, environmental risk factors may have a much larger impact on CV events than currently appreciated (17). Limited studies have examined the impact of personal protective aids on people with hypertension and diabetes, especially in India $(12,14,18)$. We therefore hypothesize that the use of personal protective aids can decrease systolic blood pressure in people with hypertension and decrease fasting blood glucose in those with diabetes. Identifying strategies that can mitigate personal risk from pollution is of major public health importance in India, considering the large number of people with hypertension and diabetes.

We therefore are conducting a randomized cross over study in Dalkhola, West Bengal to determine if people exposed to high levels of air pollution, who have hypertension or diabetes, may benefit from use of personal protective aids.

\section{Method/design:}

The study protocol received ethics approval from the institutional review board at Medstar Washington Hospital Center in Washington DC, USA and Indian Institute of Technology, Kanpur, India. The trial was registered in the clinicaltrials.gov database on April 22, 2021, and the registration number is NCT04854187. All trial participants provided written informed consent. 
The study is being conducted in a single site, the town of Dalkhola in West Bengal, India (Fig. 1). Dalkhola town has a population of around 20,000 people, with the economy largely revolving around agriculture.

This is a prospective, randomized cross over study in which participants will act as their own control. Participants between the age of 18 and 70 years, who have hypertension and/or diabetes, will be eligible for the study. (Details of inclusion and exclusion criteria are described in Table 1). After screening and obtaining informed consent, participants will be randomized to either the intervention or control arm after a two-day run-in period.

Hypertension will be diagnosed using the American College of Cardiology (ACC) 2019 guidelines of systolic blood pressure (SBP) $>130 \mathrm{mmHg}$ or diastolic blood pressure (SBP) of $>90 \mathrm{mmHg}$. Whereas diabetes will be diagnosed using the American Diabetes Association (ADA) definition of either two of the following: $\mathrm{HgA} 1 \mathrm{c} \geq 6.5$, random fasting glucose $\geq 200 \mathrm{mg} / \mathrm{dL}$ with symptoms of hyperglycemia or fasting blood glucose level $\geq 126 \mathrm{mg} / \mathrm{dL}$.

Study procedure

Screening, recruitment and randomization:

Participants will be randomized using sealed, opaque envelopes. The study outline is described further in Fig. 2.

Intervention arm:

The intervention arm will be for 4 weeks. Blood pressure and fasting blood glucose will be recorded for all participants on day 0 , day 14 and at the end of the intervention (day 28). Participants in the intervention group will be asked to use an indoor air purifier (Atlanta Healthcare 7-Stage 43-Watt Air Purifier - Fig. 3) daily for 4 weeks between the hours of 8 PM and $8 \mathrm{AM}$. The purifier will be placed in their bedroom or in the room where participants sleep at night. When the participants are outdoors (commuting, working outdoors, running errands, etc.), they will be asked to use a N95 mask (PureMe Reusable N95 AntiPollution Mask - Fig. 3). It is a reusable mask which can be washed by the participants. Every 2 weeks, the filter of the mask will be replaced, and the filter of the indoor air purifier will be washed. Study personnel will ensure that the patient remains blinded to study arm assignment during this maintenance visit.

Washout period:

At the end of the first study arm period (control or intervention), participants will have a washout period of 2 weeks, after which they will cross over to the other group for the subsequent 4 weeks.

Control arm:

The control arm will be for 4 weeks. Blood pressure and fasting blood glucose will be recorded similarly as in the intervention group on day 0 , day 14 and day 28 . The participant will be provided an identical air 
purifier and a N-95 mask, with the filter removed. At the end of two weeks, the study personnel will make dummy adjustments to the mask and indoor air purifier, to maintain blinding of the participant.

Sample size and statistical analysis

Sample size calculations are summarized in Table 2. Based on these calculations, 128 participants ( $80 \%$ power; alpha level of 0.05 ; to detect a difference of $2 \mathrm{mmHg}$; assumed SD 8) with hypertension will be randomly exposed to one of two arms for 4 weeks, followed by exposure to the other arm. Similarly, 33 participants ( $80 \%$ power; alpha level of 0.05 ; to detect a difference of $10 \mathrm{mg} / \mathrm{dL}$; estimated SD 20 ) with diabetes will be randomly exposed to one of two arms for 4 weeks, followed by exposure to the other arm.

Measurement

Pollution measurement and recording:

Given that there is no pollution monitoring unit in or near Dalkhola, we plan to directly record the pollution level in Dalkhola. We will be using Airveda Outdoor PM2510THWP monitor (19) to record ambient air pollution as average PM 2.5 levels. For indoor air pollution level recordings will include average PM 2.5 level, average PM 10 level, peak PM 2.5 level, trough PM 2.5 level, average temperature and average humidity. Indoor air pollution level will be recorded using Airveda PM2510CTH monitor (20). We will not be measuring personal exposure to PM 2.5 levels due to cost limitation.

Blood pressure measurement:

The blood pressure will be measured using an electronic BP machine (OMRON® Automatic Blood Pressure Monitor HEM-7120 - (21)). The average of the last two of three left-arm blood pressure measurements after a minimum of 5 minutes of rest with the participants seated with feet on the floor and arm supported at heart level will be used. All blood pressure measurements will be performed between $7 \mathrm{AM}$ and $10 \mathrm{AM}$.

Fasting blood glucose measurement:

The capillary blood glucose level will be measured using handheld blood glucose meters (Accu-Chek® Performa (22)).

Data Management

All data in the screening questionnaire will be entered by the data entry operator in pre-designed database forms in a RedCAP database. Analysis will be done on an intention to treat basis. Marginal (GEE) models will be used to analyze the data.

\section{Outcome Assessment:}

Primary outcome of the study will be assessment of: 
1. Absolute change in systolic blood pressure for people with hypertension using air purifiers and facemasks

2. Absolute change in fasting blood glucose for people with diabetes using air purifiers and facemasks

3. To ensure blinded outcome assessment, a dedicated study personnel who is blinded will perform the assessment.

\section{Discussion}

Our study has several strengths. To our knowledge, this is the first randomized controlled trial to evaluate personal protective aids as a therapeutic measure in people with hypertension and diabetes exposed to high levels of PM2.5 in LMIC. Our intervention is pragmatic, ensuring that if it is effective, it could potentially be of therapeutic utility to a large population.

Given the high burden of air pollution in LMICs, there is an urgent need for adaptation measures targeting people at high risk of mortality from exposure. The results of our study will demonstrate if personal protective aids can be a viable adaptation measure, for people living with hypertension and diabetes in areas with a high burden of air pollution. In addition, it will help fill knowledge gaps regarding the robustness of the association between air pollution and hypertension and diabetes. To ensure maximal co-benefit from addressing climate change and air pollution, urgent action is needed from governments in LMIC such as India. However, adequate attention also needs to be paid to adaptation measures for people living in areas of high PM2.5 exposure, who are at elevated risk for cardiovascular events. This study will fill an important knowledge gap in this regard. (23)

\section{Limitations}

Our study has a few limitations. First, we excluded people who use tobacco or a polluting cooking fuel such as an indoor wood burning stove, to ensure similar pollution exposure levels across individuals. However, this may limit the generalizability of our findings. Second, we will not measure personal exposure to PM2.5, due to logistical reasons. However, measurement of indoor and outdoor PM2.5 will provide a surrogate measure of PM2.5 exposure. We are also unable to assure consistent use of N95 masks when participants are outside of the home, however as this study was performed during the COVID-19 pandemic, this may have encouraged more consistent use of masks due to local laws and regulations.

\section{Declarations}

Ethics approval and consent to participate: IIT-K IEC Committee approval, IITK/IEC/2020-21/II/31 Consent for publication: Yes, all authors agree to publication Availability of data and materials: Data supporting our findings can be found through the corresponding author: Dweep Barbhaya (dweepbarbhaya@gmail.com) Competing interests: None 
Funding: The work is supported by the Sehat Charitable Trust, Dalkhola, India and MedStar Health Graduate Medical Education, Washington, DC, USA.

Authors' contributions

I. Conception and design: DB, AK, JT, AG

II. Administrative support: AK, DB

III. Collection and assembly of data: AK, DB

IV. Data analysis and interpretation: N/A

V. Manuscript writing: All authors

VI. Final approval of manuscript: All authors

Acknowledgements: NA

Clinical Trial Registration

Clinicaltrials.gov Identifier: NCT04854187

Available from: https://clinicaltrials.gov/ct2/show/NCT04854187

\section{References}

1. Landrigan PJ, Fuller R, Acosta NJR, Adeyi O, Arnold R, Basu N (Nil), et al. The Lancet Commission on pollution and health. The Lancet. 2018 Feb 3;391(10119):462-512.

2. Cohen AJ, Brauer M, Burnett R, Anderson HR, Frostad J, Estep K, et al. Estimates and 25-year trends of the global burden of disease attributable to ambient air pollution: an analysis of data from the Global Burden of Diseases Study 2015. The Lancet. 2017 May 13;389(10082):1907-18.

3. Global Action Plan for the Prevention and Control of NCDs 2013-2020 [Internet]. [cited 2021 Apr 10]. Available from: https://www.who.int/publications-detail-redirect/9789241506236

4. Hänninen Otto, Knol Anne B., Jantunen Matti, Lim Tek-Ang, Conrad André, Rappolder Marianne, et al. Environmental Burden of Disease in Europe: Assessing Nine Risk Factors in Six Countries. Environ Health Perspect. 2014 May 1;122(5):439-46.

5. Al-Kindi SG, Brook RD, Biswal S, Rajagopalan S. Environmental determinants of cardiovascular disease: lessons learned from air pollution. Nat Rev Cardiol. 2020 Oct;17(10):656-72.

6. Rajagopalan S, Al-Kindi SG, Brook RD. Air Pollution and Cardiovascular Disease: JACC State-of-theArt Review. J Am Coll Cardiol. 2018 Oct 15;72(17):2054-70.

7. Prüss-Üstün A, Wolf $J$ de, Corvalán CF, Bos R, Neira MP. Preventing disease through healthy environments: a global assessment of the burden of disease from environmental risks. Geneva, Switzerland: World Health Organization; 2016. 147 p. 
8. Allen RW, Carlsten C, Karlen B, Leckie S, Eeden S van, Vedal S, et al. An Air Filter Intervention Study of Endothelial Function among Healthy Adults in a Woodsmoke-impacted Community. Am J Respir Crit Care Med. 2011 May 1;183(9):1222-30.

9. Weichenthal S, Mallach G, Kulka R, Black A, Wheeler A, You H, et al. A randomized double-blind crossover study of indoor air filtration and acute changes in cardiorespiratory health in a First Nations community. Indoor Air. 2013;23(3):175-84.

10. Indoor Particles Affect Vascular Function in the Aged | An Air Filtration-based Intervention Study | American Journal of Respiratory and Critical Care Medicine [Internet]. [cited 2020 Mar 22]. Available from: https://www.atsjournals.org/doi/full/10.1164/rccm.200704-6320C?url_ver=Z39.882003\&rfr_id=ori:rid:crossref.org\&rfr_dat=cr_pub\%3dpubmed

11. Langrish JP, Mills NL, Chan JK, Leseman DL, Aitken RJ, Fokkens PH, et al. Beneficial cardiovascular effects of reducing exposure to particulate air pollution with a simple facemask. Part Fibre Toxicol. 2009 Mar 13;6:8.

12. Chen R, Zhao A, Chen H, Zhao Z, Cai J, Wang C, et al. Cardiopulmonary Benefits of Reducing Indoor Particles of Outdoor Origin: a Randomized Double-Blind Crossover Trial of Air Purifiers. J Am Coll Cardiol. 2015 Jun 2;65(21):2279-87.

13. Shi J, Lin Z, Chen R, Wang C, Yang C, Cai J, et al. Cardiovascular Benefits of Wearing ParticulateFiltering Respirators: A Randomized Crossover Trial. Environ Health Perspect. 2017 Feb;125(2):17580.

14. Li Huichu, Cai Jing, Chen Renjie, Zhao Zhuohui, Ying Zhekang, Wang Lin, et al. Particulate Matter Exposure and Stress Hormone Levels. Circulation. 2017 Aug 15;136(7):618-27.

15. Münzel T, Sørensen M, Gori T, Schmidt FP, Rao X, Brook J, et al. Environmental stressors and cardiometabolic disease: part I-epidemiologic evidence supporting a role for noise and air pollution and effects of mitigation strategies. Eur Heart J. 2017 Feb 21;38(8):550-6.

16. Münzel T, Sørensen M, Gori T, Schmidt FP, Rao X, Brook FR, et al. Environmental stressors and cardiometabolic disease: part II-mechanistic insights. Eur Heart J. 2017 Feb 21;38(8):557-64.

17. Environmental stressors and cardio-metabolic disease: part II-mechanistic insights | European Heart Journal | Oxford Academic [Internet]. [cited 2020 Mar 22]. Available from: https://academic.oup.com/eurheartj/article/38/8/557/3056928

18. Chuang H-C, Ho K-F, Lin L-Y, Chang T-Y, Hong G-B, Ma C-M, et al. Long-term indoor air conditioner filtration and cardiovascular health: A randomized crossover intervention study. Environ Int. 2017 Sep 1;106:91-6.

19. Airveda High Accuracy Outdoor Air Quality Monitor [Internet]. [cited 2021 Apr 10]. Available from: https://airveda.com/outdoor-air-quality-monitor

20. Airveda High Accuracy CO2, PM2.5, PM10, Temp, Humidity Smart Laser Air Quality Monitor - Wi-Fi enabled, App-Enabled [Internet]. [cited 2021 Apr 10]. Available from: https://airveda.com/airveda-pmco2-air-quality-monitor 
21. HEM-7120 - Blood Pressure Monitors (Upper Arm) - Omron Healthcare India [Internet]. [cited 2021 Apr 10]. Available from: https://www.omronhealthcare-ap.com/in/product/12-hem-7120

22. Accu-Chek Performa [Internet]. Accu-Chek®. [cited 2021 Apr 10]. Available from: https://www.accuchek.in/meter-systems/performa

23. Markandya A, Sampedro J, Smith SJ, Dingenen RV, Pizarro-Irizar C, Arto I, et al. Health co-benefits from air pollution and mitigation costs of the Paris Agreement: a modelling study. Lancet Planet Health. 2018 Mar 1;2(3):e126-33.

\section{Tables}

Due to technical limitations, tables are only available as a download in the Supplemental Files section.

\section{Figures}


Figure 1: Map showing study site in Dalkhola, West Bengal

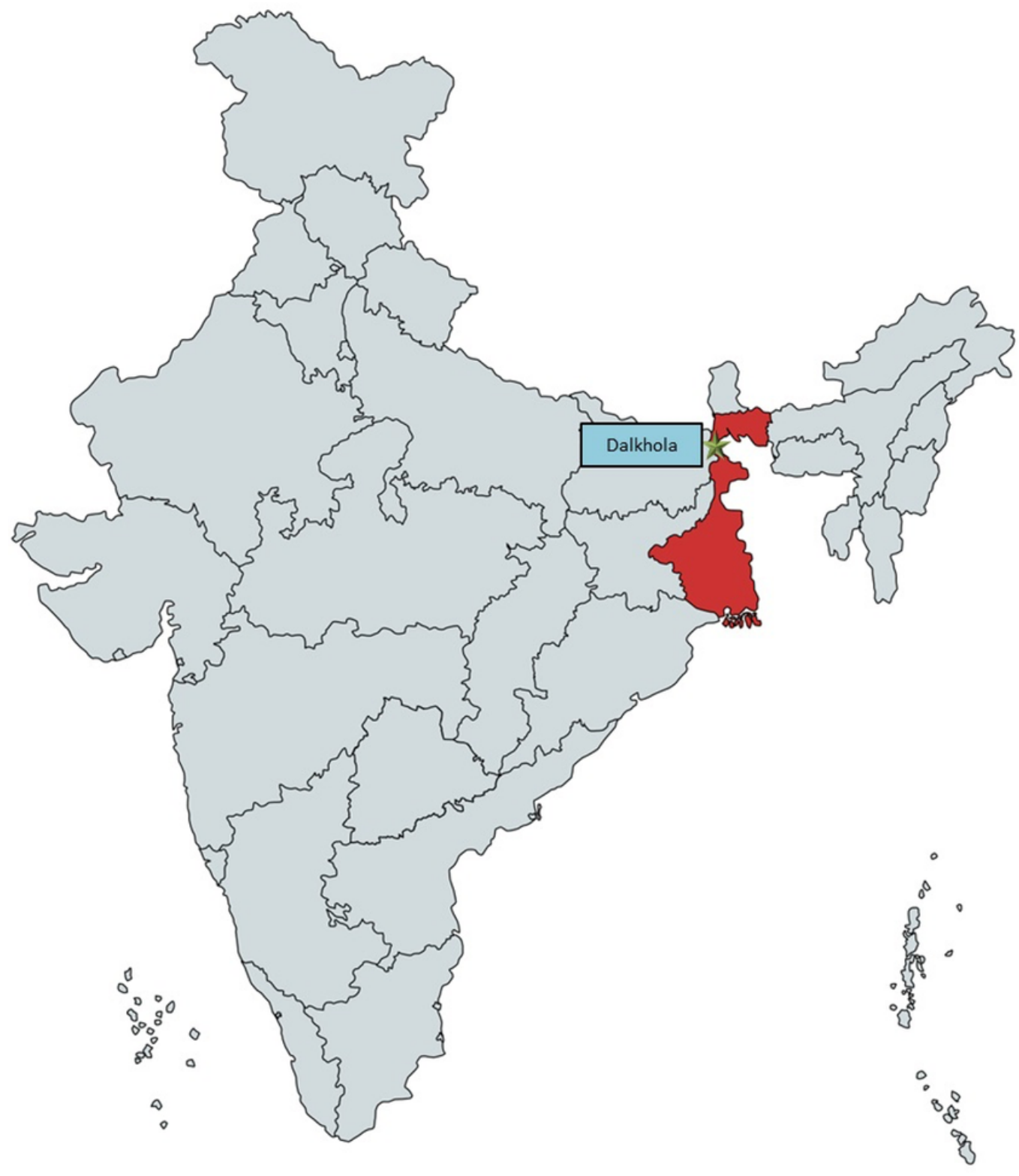

Figure 1

See image above for figure legend 


\section{Figure 2: Study Outline}

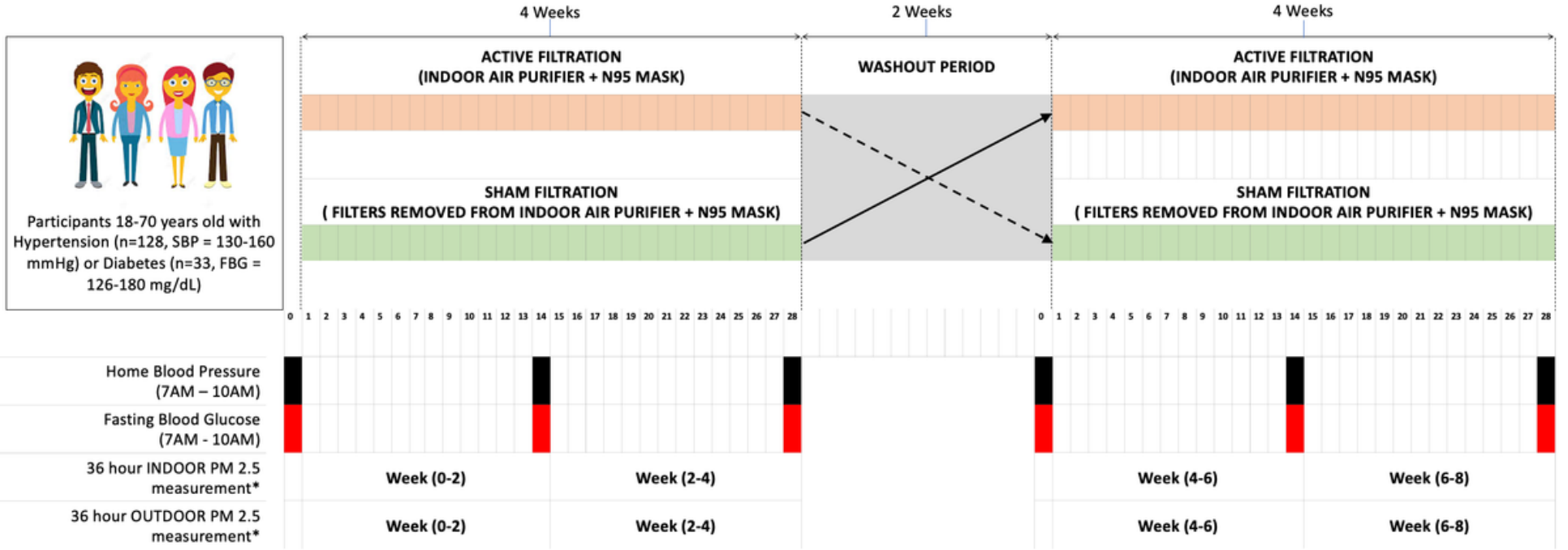

* Only subset of patients will undergo PM 2.5 monitoring

\section{Figure 2}

See image above for figure legend 
Figure 3: N95 mask with filters (A - PureMe Reusable N95 Anti-Pollution Mask) and indoor air purifier (B - Atlanta Healthcare 7-Stage 43-Watt Air Purifier)
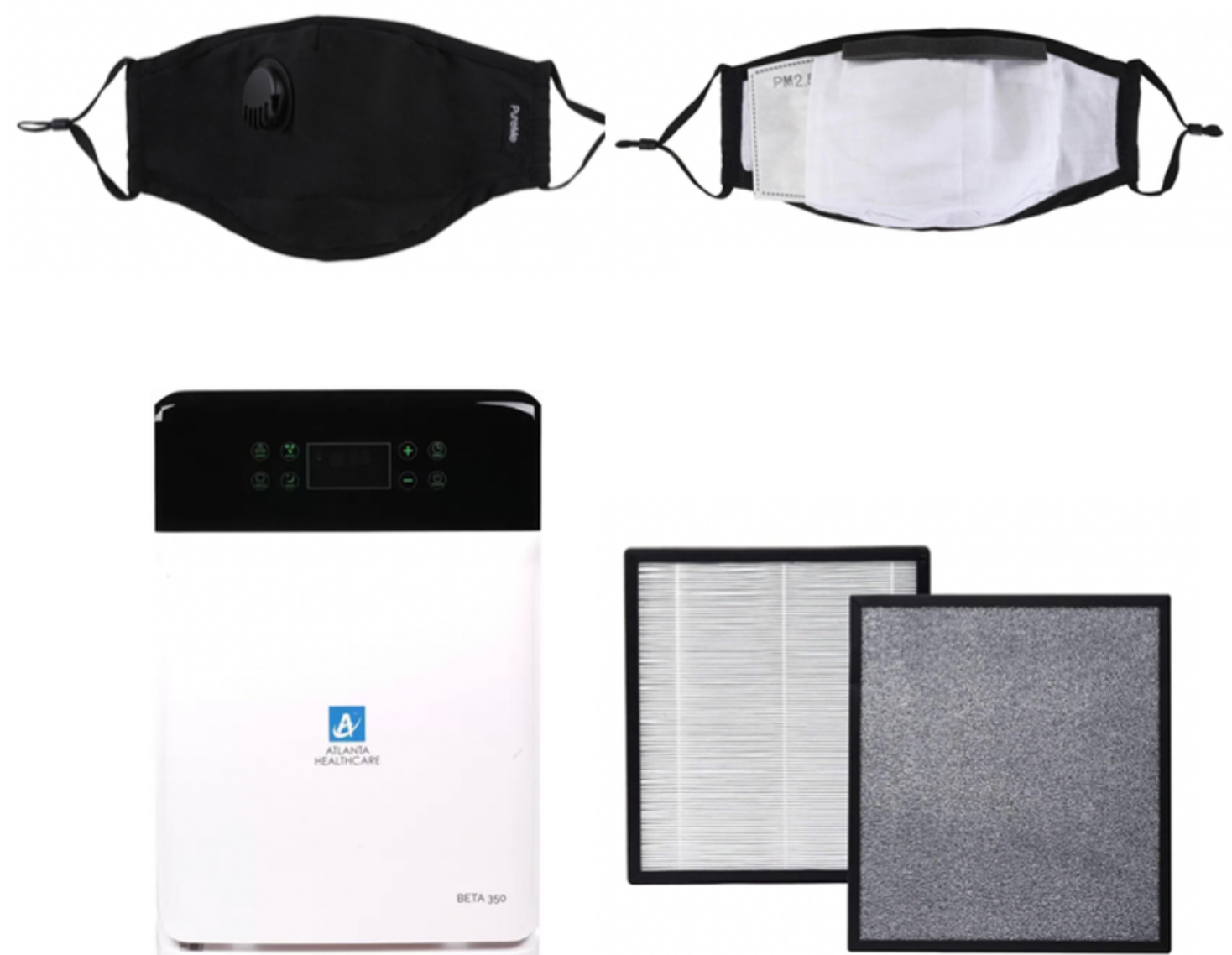

Figure 3

See image above for figure legend

\section{Supplementary Files}

This is a list of supplementary files associated with this preprint. Click to download.

- Table1.png

- Table2.png 